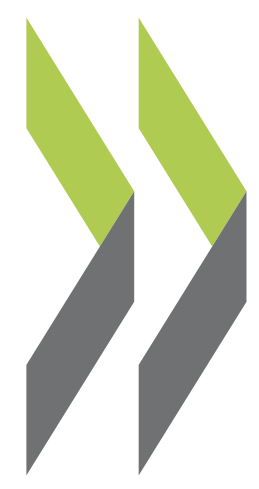

Documents de travail de l'OCDE sur la science, la technologie et l'industrie 1997/02

Révision des classifications des secteurs et des produits de haute technologie

\section{Thomas Hatzichronoglou}


REVISION DES CLASSIFICATIONS DES SECTEURS ET DES PRODUITS DE HAUTE TECHNOLOGIE

\title{
Thomas Hatzichronoglou
}

\section{ORGANISATION DE COOPERATION ET DE DEVELOPPEMENT ECONOMIQUES}

\author{
Paris
}

59868

Document complet disponible sur OLIS dans son format d'origine

Complete document available on OLIS in its original format 


\section{DOCUMENTS DE TRAVAIL DE LA DSTI}

La série de Documents de travail de la Direction de la science, de la technologie et de l'industrie a été créée dans le but de rendre accessibles à un plus large public les analyses rédigées par des membres de la Direction ou par des consultants externes travaillant sur des projets pour l'OCDE. Les rapports sont d'une nature technique et/ou analytique et traitent de questions sur les données, la méthodologie et l'analyse empirique dans les domaines de travail de la Direction. Les Documents de travail sont en général disponibles uniquement dans leur langue d'origine - anglais ou français - et présentent un bref résumé dans l'autre langue.

Des commentaires sur ces Documents seraient appréciés et pourront être adressés à l'OCDE, 2 rue André Pascal, 75775 Paris Cedex 16, France.

Les opinions exprimées sont celles des auteurs et ne reflètent pas nécessairement celles de l'OCDE ni celles des gouvernements des pays Membres.

\section{Copyright OCDE, 1997}

Les demandes de reproduction ou de traduction doivent être adressées à :

M. le Chef du Service des Publications, OCDE, 2 rue André Pascal, 75775 Paris, Cedex 16, France. 


\title{
REVISION OF THE HIGH-TECHNOLOGY SECTOR AND PRODUCT CLASSIFICATION
}

\author{
Thomas Hatzicronoglou
}

This paper describes the methods used to classify the OECD countries' industrial sectors and manufactures by level of technology, and presents the resulting classifications. In the proposed new classification by industrial sector, the concept of technology intensity has been expanded to take into account both the level of technology specific to the sector (measured by the ratio of R\&D expenditure to value added) and the technology embodied in purchases of intermediate and capital goods. Four groups of industries have been identified on the basis of the degree of technology intensity.

The classification by product consists solely of high-technology products (products which are the most technology-intensive). The classification was drawn up by the OECD Secretariat in collaboration with Eurostat, the object being to finalise the approach by sector and provide a more appropriate instrument for analysing international trade. Because no detailed data were available for services, the two proposed classifications concern only manufacturing industry.

\section{RÉVISION DES CLASSIFICATIONS DES SECTEURS ET DES PRODUITS DE HAUTE TECHNOLOGIE}

\section{Thomas Hatzicronoglou}

Ce document expose les méthodes pour classer les secteurs industriels et les produits manufacturés des pays de l'OCDE selon leur intensité technologique et présente les classifications qui en résultent. Dans la nouvelle classification proposée par secteur industriel, la notion d'intensité technologique a été élargie pour tenir compte à la fois de l'effort technologique propre au secteur (mesuré par le ratio des dépenses de R-D sur la valeur ajoutée) et de la technologie incorporée dans les achats de biens intermédiaires et d'équipement. Quatre groupes d'industries ont été identifiés en fonction du niveau de leur intensité technologique.

La classification par produit se réfère exclusivement aux produits de haute technologie (produits dont l'intensité technologique est la plus élevée). Cette classification a été élaborée par le Secrétariat de l'OCDE en coopération avec Eurostat, afin de compléter l'approche par secteur et fournir un instrument plus approprié pour l'analyse des échanges internationaux. Du fait de la non disponibilité de données détaillées sur les services, les deux classifications proposées concernent exclusivement l'industrie manufacturière. 


\section{INTRODUCTION}

Dans le contexte de mondialisation de l'économie, la technologie est un facteur clé pour améliorer la croissance et la compétitivité des entreprises.

Les firmes dont l'effort technologique est plus intensif, innovent davantage, gagnent de nouveaux marchés, utilisent les ressources disponibles de manière plus productive et offrent des rémunérations généralement plus élevées aux personnes qu'elles emploient. Les industries appelées de "haute technologie" sont celles qui connaissent la plus forte expansion dans le commerce international et leur dynamique contribue à l'amélioration des performances d'autres secteurs (effets "spillovers"). Pour pouvoir analyser les effets de ces technologies sur la performance industrielle, il est important de pouvoir identifier les industries et les produits qui sont technologiquement les plus intensifs, à travers des critères permettant la construction de classifications spécifiques harmonisées au plan international.

Le présent document a comme objectif d'exposer les méthodes utilisées par le Secrétariat de l'OCDE pour classer les secteurs et les produits selon leur intensité technologique et de présenter les classifications qui en ont résulté.

Les premiers travaux analytiques du Secrétariat de l'OCDE en matière de haute technologie, concernant notamment les échanges ${ }^{1}$ étaient effectués à partir de la classification réalisée aux Etats-Unis qui ensuite était appliquée à l'ensemble des pays de l'OCDE. Si cette approche permettait pour la première fois, dans le domaine de haute technologie, de situer les pays les uns par rapport aux autres, elle présentait l'inconvénient d'extrapoler à l'ensemble de la zone OCDE la structure de l'industrie américaine. C'est la raison pour laquelle dans une seconde phase le Secrétariat a mis au point en 1989 une première classification sur la base d'un échantillon comprenant 11 pays. Cette classification était fondée sur le critère d'intensité directe en R-D (dépenses de R-D par rapport à la production) pondéré par secteur et pays. Cette classification a donné lieu à l'élaboration d'une liste regroupant les industries en trois catégories ${ }^{2}$ (haute, moyenne et faible technologie) largement utilisée par les pays Membres de l'OCDE ainsi que par de nombreux pays non membres.

Elle avait l'avantage d'offrir pour les comparaisons internationales un instrument simple et cohérent, mais en même temps elle comportait des limites liées en grande partie à l'absence de données sectorielles suffisamment désagrégées. Dix ans après l'élaboration de la première liste des secteurs classés en fonction de leur intensité technologique il était devenu nécessaire de faire le point sur l'expérience de cette période et envisager certaines améliorations. Celles-ci prennent mieux en compte l'évolution technologique en utilisant des données beaucoup plus récentes, et en intégrant différents aspects de la diffusion de la technologie (intensités indirectes). Ainsi, le Secrétariat de l'OCDE a été amené à élaborer deux nouvelles listes: une liste concernant les industries manufacturières (approche sectorielle) et une seconde liste concernant les produits manufacturés (approche par produit).

Les données utilisées pour mettre au point la liste par secteur est fondée sur la classification CITI Révision 2 (Classification internationale type de l'industrie). A partir de cette liste la nouvelle classification obtenue ne concerne que l'indutrie manufacturière pour laquelle le Secrétariat disposait de 
séries longues et relativement complètes. Toutefois, dans l'approche sectorielle, il sera à terme, nécessaire d'intégrer également les services dès que la disponibilité des données le permettra, dans la mesure où ceux-ci, d'utilisateurs intensifs de technologie, en deviennent de plus en plus producteurs.

L'approche par produit a été mis au point plus récemment pour compléter l'approche sectorielle et fournir un instrument plus appropriée pour l'analyse des échanges internationaux. Elle est fondée sur la classification CTCI Révision 3 (classification type du commerce international).

\section{L'approche sectorielle}

L'élaboration d'une classification exhaustive des industries selon leur intensité technologique comporte plusieurs difficultés. La première, concerne la définition des critères qui permettent d'identifier le contenu technologique d'une industrie. Une seconde difficulté concerne le concept sous-jacent de ce qu'est une industrie de haute technologie : est-ce une industrie qui produit de la technologie, ou bien est-ce une industrie qui en fait un usage intensif ?. Une troisième réside dans le fait qu'il existe toujours un certain degré d'arbitraire dans le choix des seuils qui délimitent les différents groupes d'industries de la classification.

Pour identifier le contenu technologique d'une industrie, le Secrétariat a adopté différents critères mais leur quantification a buté sur l'absence de données disponibles. Ainsi, le seul critère retenu a été l'intensité de R-D ${ }^{3}$.

Pour surmonter la seconde difficulté, il a fallu comparer les mesures des intensités de R-D directes et indirectes. Deux indicateurs d'intensités directes ont été utilisées et un indicateur concernant les intensités globales de R-D (somme des intensités directes et indirectes). Les deux indicateurs d'intensité ont été établies pour chacun des 22 secteurs manufacturés de 10 pays de l'OCDE ${ }^{4}$ tandis que la liste OCDE a été obtenue en pondérant chaque secteur par son poids dans la production ou la valeur ajoutée de l'ensemble des 10 pays en utilisant comme taux de change les parités de pouvoir d'achat du PIB. Dans le cas des intensités globales directes, les intensités ont été calculées de la même façon que précédemment. Quant aux intensités indirectes, il a fallu tenir compte de la technologie (dépenses de R-D) incorporée dans les biens intermédiaires et les biens en capital achetés sur le marché intérieur ou importés. Ainsi, la technologie passe d'une industrie (et d'un pays) à l'autre lorsque l'industrie à l'origine de la R-D vend ses produits incorporant sa R-D à d'autres industries qui les utiliseront comme inputs dans le processus de production. Pour cela, on a eu recours aux coefficients techniques des industries manufacturières à partir de matrices input-output. En supposant que les proportions des dépenses de R-D incorporées dans la production pour un type d'input donné et pour tous les groupes de produits demeuraient constantes, les coefficients input-ouptut ont été multipliés par les intensités directes de R-D (voir aussi annexe 1).

Ces indicateurs ont été calculés sur une longue période (1973-92) mais la classification finale a été construite pour les données 1980 et 1990 à partir de ces indicateurs de façon à ce qu'elle reste stable pour chacun d'entre eux, à savoir pour chaque indicateur, les industries classées dans une catégorie supérieure avaient une intensité moyenne OCDE plus élevée que les industries classées dans une catégorie inférieure à l'exception du secteur du pétrole (voir plus loin). Ainsi, de ces calculs, quatre groupes d'industries manufacturières ont été identifiés : ceux de i) haute technologie, de ii) moyenne-haute technologie, de iii) moyenne-faible technologie et de $i v$ ) faible technologie.

La prise en compte dans les calculs des intensités indirectes ne modifie guère l'appartenance de chaque industrie à chacun des quatre groupes précédents, mais elle peut modifier son rang. Cela met en évidence le fait que les industries qui consacrent une part élevée de leur chiffre d'affaires ou de leur 
production à la R-D, utilisent également les équipements et les biens intermédiaires les plus avancés technologiquement. Ce résultat montre que pour ces industries il existe une forte corrélation de rangs entre intensités directes (production de technologie) et intensités indirectes (utilisation de technologie).

Enfin, les seuils adoptés pour regrouper les industries en 4 catégories sont moins arbitraires qu'ils n'y paraissent. Les industries appartenant à un groupe ont des intensités de R-D supérieures à celles du groupe inférieur sur une longue période (1980-92).

La distinction entre les groupes de "moyenne-haute" et de "moyenne-faible" ainsi que entre ceux de "moyenne-faible" et de "faible technologie" est plus évidente lorsque les intensités de R-D sont calculées par rapport à la production que par rapport à la valeur ajoutée, mais dans les deux cas, les seuils choisis assurent une stabilité dans le temps et une stabilité médiane à travers des pays, à savoir les industries classées dans une catégorie supérieure ont une intensité médiane supérieure aux industries appartenant à une catégorie inférieure.

La liste ainsi adoptée (tableau 1 et annexe 2, tableau 3) correspond aux intensités de R-D globales (directes et indirectes) et elle reste stable sur toute la période de référence. Elle diffère de la liste utilisée jusqu'en 1994 sur trois points :

- Premièrement, l'intensité technologique du secteur des machines électriques s'est affaiblie tandis que celle des instruments scientifiques a augmenté et même, à partir de 1986, a tendance à se rapprocher des intensités des secteurs de haute technologie. Lorsque les intensités sont calculées par rapport à la valeur ajoutée, le secteur des instruments scientifiques apparait moins intensif que celui des véhicules automobiles mais il est plus intensif si les calculs sont effectués par rapport à la production. Pour assurer une stabilité dans le temps, on a décidé de détacher les instruments scientifiques du groupe de haute technologie ainsi que les machines électriques, ces deux industries font partie désormais du groupe de moyenne-haute technologie.

- Deuxièmement, à l'intérieur du groupe de moyenne technologie, on distingue deux sous-catégories, celle de moyenne-haute, et de moyenne faible technologie.

- Troisièmement, certains secteurs classés auparavant dans le groupe de faible-technologie font désormais partie de la moyenne faible-technologie du fait que leur intensité a augmenté. C'est le cas notamment de la construction navale. En revanche, d'autres secteurs ont été reclassés du fait qu'ils existent maintenant 4 groupes d'industries au lieu de trois auparavant. C'est le cas des métaux ferreux et non ferreux, des fabrications métalliques et du raffinage du pétrole. L'intensité de cette dernière industrie est même supérieure à celle des machines électriques si celle-ci est calculée par rapport à la valeur ajoutée et non pas par rapport à la production. Ce cas constitue d'ailleurs la seule exception concernant le critère de stabilité et elle est due sans doute à la forte proportion de consommation intermédiaire qui caractérise ce secteur. On constate également que l'intensité du caoutchouc et des matières plastiques a baissé au cours des vingt dernières années, tandis que celle des autres matériels de transport a augmenté, se rapprochant à celle des machines électriques. 
Tableau 1. Industries manufacturières classées selon leur intensité technologique globale (CITI Révision 2)

\section{Haute technologie}

1. Aérospatiale

2. Ordinateurs, machines de bureau

3. Electronique-communications

4. Pharmaceutique

\section{Moyenne-haute technologie}

5. Instruments scientifiques

6. Véhicules automobiles

7. Machines et appareils électrique

8. Industries chimiques

9. Autres matériels de transport

10. Machines non électriques

\section{Moyenne-faible technologie}

11. Caoutchouc et matières plastiques

12. Construction navale

13. Autres industries manufacturières

14. Métaux non ferreux

15. Produits minéraux non métalliques

16. Ouvrages en métaux

17. Raffinage du pétrole

18. Métaux ferreux

\section{Faible technologie}

19. Papier, imprimerie et édition

20. Textile, habillement et cuir

21. Alimentation, boissons et tabac

22. Bois et meubles

\section{CITI Révision 2}

3845

3825

3832

3522

385

3843

383-3832

$351+352+3522$

$3842+3844+3849$

382-3825
34

32

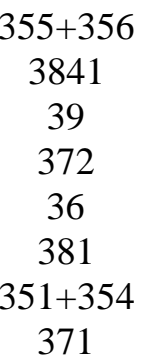

31

33

\section{L'approche par produit}

L'approche par produit complète l'approche sectorielle et ouvre la voie à des analyses beaucoup plus détaillées dans le domaine des échanges et de la compétitivité.

Cette approche se distingue de l'approche sectorielle au moins sur trois points.

Tandis qu'une industrie peut être technologiquement très intensive dans un pays et faiblement intensive dans un autre, il est difficile à admettre que le même produit puisse être classé à la haute technologie dans certains pays et dans la moyenne ou la faible technologie dans d'autres. Si tel était le cas, c'est qu'il s'agit en principe de produits différents. ${ }^{5}$. De ce fait, l'existence de listes nationales parallèlement à la liste OCDE ne peut se justifier que dans le cas ou au niveau national, la liste des produits de haute technologie est beaucoup plus désagrégée. 
Deuxièmement, l'approche par produit prend en compte certains produits qui en général sont exclus de la liste par secteur, du fait qu'ils sont fabriqués par des secteurs de moyenne technologie. Par ailleurs, elle permet de mieux évaluer la proportion réelle de haute technologie des secteurs, dans la mesure où dans l'approche par produit sont exclus tous les produits qui n'appartiennent pas à la haute technologie même s'ils sont fabriqués par des industries de haute technologie.

Une troisième spécificité de l'approche par produit est qu'elle concerne les produits appartenant exclusivement à la haute technologie. L'approche choisie ne permet pas pour l'instant de distinguer les produits de moyenne-haute, moyenne-faible ou faible intensité technologique, au moins pas au niveau d'agrégation qui a été choisi.

En 1994, une première liste était préparée par le Secrétariat en coopération avec l'Institut Fraunhofer en Allemagne et correspondait à la classification CTCI Révision 3 du Commerce extérieur à 3 chiffres. Cette liste était le résultat de calculs concernant les intensités de R-D par groupe de produit (dépenses de R-D/chiffre d'affaires) pour lesquels 6 pays étaient pris en considération (Etats-Unis, Japon, Allemagne, Italie, Suède et Pays-Bas).

Dans l'approche par produit, le nombre de pays pris en compte n'a pas beaucoup d'importance, contrairement à l'approche sectorielle, étant donné que l'appartenance d'un produit à la haute technologie est indépendante de considérations nationales et par conséquent pour un niveau d'agrégation donné une liste de produits de haute technologie peut être établie à partir d'un nombre plus restreint de pays.

La liste proposée par le Secrétariat en 1994 constituait un premier effort important dans ce nouveau domaine et a servi comme base aux travaux qui ont suivi et qui ont abouti à la liste présentée au tableau 2. Ces travaux complémentaires étaient motivés par trois problèmes que présentait la liste en question.

En premier lieu, le niveau d'agrégation à 3 chiffres s'il constitue une amélioration importante par rapport au niveau de l'approche sectorielle, reste encore assez limité. Probablement l'inconvénient le plus important était la description des produits à 4 et 5 chiffres appartenant aux groupes de produits précédents. De toute évidence à ce niveau d'agrégation, de nombreux produits ne pouvaient pas justifier leur appartenance à la haute technologie, et dans les travaux qui ont suivi il a fallu les exclure. Dans ce but, lorsque des doutes subsistaient pour certains produits, on a eu recours à l'avis d'experts de ces produits.

Deuxièmement, l'industrie automobile était classée globalement dans la haute technologie. Or, cette industrie dans l'approche sectorielle appartient à la moyenne-haute technologie et il serait difficile de justifier pour les automobiles un traitement global différent selon que l'on adopte l'approche par secteur ou l'approche par produit. Par ailleurs, le poids considérable des automobiles dans les échanges internationaux modifierait de manière radicale le profil des pays. C'est pour ces raisons que nous avons préféré exclure l'automobile de la liste de produits de haute technologie.

Troisièmement, en dépit des calculs, le contenu technologique de certains produits appartenant à des secteurs de moyenne et de faible technologie même à un niveau plus désagrégé, n'était pas confirmé par l'avis d'experts. Face à ce dilemme, nous avons préféré les exclure de la liste de produits de haute technologie.

Ainsi, la liste proposée au tableau 2 est relativement compatible avec les listes des secteurs dans la mesure où les produits ont été classés selon leur secteur d'appartenance ${ }^{6}$. Elle est plus restrictive que la précédente liste par produit et bien davantage que les listes sectorielles. En revanche, elle comprend certains produits fabriqués par les industries de moyenne-haute technologie. 
Une nouvelle banque de données par produit a été ainsi créée pour le commerce extérieur à partir de la liste du tableau 4 (annexe 2). Pour la première fois dans une telle banque ont été intégrées les valeurs unitaires des différents produits (valeurs/quantités) pour les exportations et les importations globales et également pour le commerce bilatéral. Des travaux supplémentaires permettront à partir des valeurs unitaires d'obtenir des estimations des gammes de produits. Pour tenir compte de ces aspects qualitatifs du commerce extérieur, il va falloir procéder en même temps à une analyse approfondie de la compétitivité de chaque pays pour chaque produit. Ces travaux devraient permettre la création de listes individuelles par pays reflétant la gamme de chaque produit échangé.

Depuis 1994, la classification du commerce extérieur CTCI Révision 3 à 5 chiffres a été remplacée par la classification du Système Harmonisé qui contient 6 chiffres. Par conséquent, la liste par produit pourrait dans un avenir proche contenir un plus grand nombre de produits.

\section{Principales limites des classifications proposées}

Sans entrer ici dans des considérations théoriques et conceptuelles concernant d'abord la mesure de la technologie elle-même et de la haute technologie ensuite, on pourrait citer quelques unes des limites qui sont directement liées à la construction des listes proposées.

\section{Tableau 2. Liste des produits de haute technologie - CTCI Révision 3 (Période 1988-95)}

\begin{tabular}{|c|c|c|}
\hline 1- & Aérospatiale & $\begin{array}{l}{[7921+7922+7923+7924+7925+79291} \\
+79293+(714-71489-71499)+87411]\end{array}$ \\
\hline $2-$ & Ordinateurs - Machines de bureau & $\begin{array}{l}{[75113+75131+75132+75134+(752} \\
-7529)+75997]\end{array}$ \\
\hline $3-$ & Electronique - Communications & $\begin{array}{l}{[76381+76383+(764-76493-76499)} \\
+7722+77261+77318+77625+77627 \\
+7763+7764+7768+89879]\end{array}$ \\
\hline 4- & Pharmacie & {$[5413+5415+5416+5421+5422]$} \\
\hline 5- & Instruments scientifiques & $\begin{array}{l}{[774+8711+8713+8714+8719+87211} \\
+(874-87411-8742)+88111+88121 \\
+88411+88419+89961+89963+89966 \\
+89967]\end{array}$ \\
\hline 6- & Machines électriques & $\begin{array}{l}{[77862+77863+77864+77865+7787} \\
+77884]\end{array}$ \\
\hline 7- & Chimie & $\begin{array}{l}{[52222+52223+52229+52269+525} \\
+531+57433+591]\end{array}$ \\
\hline 8- & Machines non électriques & $\begin{array}{l}{[71489+71499+71871+71877+71878} \\
+72847+7311+73131+73135+73142 \\
+73144+73151+73153+73161+73163 \\
+73165+73312+73314+73316+7359 \\
+73733+73735]\end{array}$ \\
\hline
\end{tabular}

9- Armement [ 891-- ]

La première limite concerne les critères utilisés. Seules les intensités de R-D, qu'elles soient directes ou indirectes ont été prises en compte. Or, si la recherche est une caractéristique extrêmement importante 
de la haute technologie elle n'est pas la seule. D'autres facteurs peuvent jouer aussi un rôle important (ex: le personnel scientifique et technique, la technologie incorporée dans les brevets, licences et know-how, les coopérations stratégiques des firmes en matière de technologie, l'obsolescence accélérée des connaissances disponibles, le renouvellement rapide des équipements, etc.).

Mais les mesures des intensités de R-D présentent aussi deux autres inconvénients. Elles défavorisent les secteurs et les périodes pendant lesquelles le chiffre d'affaires ou la production augmentent plus rapidement que les dépenses de R-D grâce à une forte croissance de la demande ou à une politique commerciale particulièrement dynamique. L'origine de cette difficulté qui affecte davantage les résultats d'un pays et d'une année particulière, est que les calculs sont effectués à partir de données sur les flux et non pas sur les stocks. En l'absence de données sur les stocks, cette difficulté a été pratiquement éliminée en prenant la moyenne OCDE pour chaque année et chaque industrie.

Plus spécialement dans l'approche par secteur, les intensités de R-D peuvent également être biaisées par le fait que dans chaque secteur toute la recherche de chaque secteur est attribuée à l'activité principale des firmes qui composent le secteur. On peut ainsi constater qu'une partie importante de la R-D de l'industrie aérospatiale concerne l'électronique, ce qui est le cas également pour d'autres secteurs. Alors l'intensité de R-D de l'industrie aérospatiale se trouvera surestimée et celle de l'électronique sous-estimée.

Une autre limite qui concerne davantage l'approche sectorielle est l'absence de données assez désagrégées. Lorsqu'il s'agit de classer 22 secteurs en fonction de leur intensité technologique, il est peu probable qu'on obtiendrait des regroupements de secteurs fondamentalement différents, notamment pour le groupe de haute technologie même si on ajoutait des critères de sélection supplémentaires.

La principale limite de l'absence de données détaillées est que de nombreux produits fabriqués par les secteurs de haute technologie sont de technologie moyenne voire faible et inversement certains produits fabriqués par les secteurs de moyenne ou de moyenne-faible technologie sont de haute technologie.

C'est en principe pour remédier à cette difficulté que l'approche par produit a été développée. Cependant, celle-ci présente également 3 autres limites. Premièrement, la sélection des produits de haute technologie ne peut être fondée exclusivement sur des méthodes quantitatives, à moins qu'on adopte un niveau d'agrégation relativement élevé. Le recours à l'avis d'experts permet sans doute d'obtenir des listes extrêmement détaillées mais il s'agit d'opérations relativement lourdes dont les résultats peuvent être difficilement reproductibles dans leur totalité par d'autres panels d'experts.

La seconde limite concerne l'absence hiérarchique entre produits. Si la sélection n'est pas fondée exclusivement sur des mesures quantitatives, il est difficile de classer les produits par ordre croissant ou décroissant qui dépend du niveau de leur contenu technologique. C'est pour contourner cette difficulté que le Secrétariat a calculé les valeurs unitaires des produits exportés et importés, et à travers une méthodologie spéciale, envisage de classer les produits en fonction de leur gamme.

Une dernière limite enfin concerne l'absence de comparabilité de ces données aux autres données industrielles. A part la nouvelle classification PRODCOM concernant les données de production, les autres variables industrielles notamment l'emploi, la valeur ajoutée ou la formation brute du capital fixe ne sont disponibles qu'au niveau des secteurs et non pas des produits. 


\section{Annexe 1}

Méthode d'évaluation des intensites de R-D indirectes

a) Estimation des tableaux des flux technologiques par pays et par année

b) Approche inverse de la matrice de Leontief 


\section{Méthode d'évaluation des intensités de R-D indirectes}

\section{a) Estimation des tableaux des flux technologiques par pays et par année}

La base de données entrées-sorties de l'OCDE $^{7}$ distingue, au sein des flux de transactions entre secteurs, les flux intérieurs et les flux d'importations. Par ailleurs, la composition par produits de la formation brute de capital fixe est ventilée par secteur d'origine et est disponible séparément pour les flux intérieurs et les importations. Sur la base de ces comptes, un modèle d'échanges inter-industriels permettant de mesurer le total de la R-D incorporée peut être décrit de la manière suivante.

\section{$R$-D incorporée dans les achats de biens intermédiaires intérieurs}

Les montants des flux de R-D incorporés dans les biens intermédiaires intérieurs achetés par le secteur $\mathrm{j}$ au secteur $\mathrm{i}, \mathrm{RII}^{\mathrm{d}}{ }_{\mathrm{ij}}$, peut être obtenu par la formule :

$$
R I I^{d}{ }_{i j}=\frac{X_{i j}^{d}}{X_{i}} \cdot R_{i}=X^{d}{ }_{i j} \frac{R_{i}}{X_{i}}
$$

où $\mathrm{X}_{\mathrm{ij}}$ représente la quantité de produits du secteur $\mathrm{i}$ achetée par le secteur $\mathrm{j}, \mathrm{X}_{\mathrm{i}}$ le total des ventes du secteur $\mathrm{i}$ et $\mathrm{R}_{\mathrm{i}}$ représente les dépenses intramuros de $\mathrm{R}-\mathrm{D}$ du secteur $\mathrm{i} . \mathrm{R}_{\mathrm{i}} / \mathrm{X}_{\mathrm{i}}$ est l'intensité de $\mathrm{R}-\mathrm{D}$ du secteur i par unité de production brute. Bien qu'il soit très important de prendre en compte les décalages temporels de la R-D (en moyenne 2 à 3 ans selon des travaux antérieurs) et de construire de meilleurs indicateurs du contenu technologique des produits (par ex. estimation du stock de R-D) on a simplement utilisé les dépenses courantes de R-D pour estimer les flux de R-D incorporée d'une année donnée. La correction de ces lacunes sera envisagée dans un avenir proche.

\section{$R-D$ incorporée dans les achats de biens d'équipement intérieurs}

De même la R-D incorporée dans les dépenses en capital du secteur $\mathrm{j}$ concernant le produit du secteur i, RINV $d_{i j}$ peut être représentée par l'équation :

$$
R I N V_{i j}^{d}=I N V_{i j}^{d} \frac{R_{i}}{X_{i}}
$$

où RINV $_{\mathrm{ij}}$ représente les ventes de biens d'équipement du secteur i au secteur $\mathrm{j}$ au cours d'une année donnée.

La tendance à la croissance des échanges de haute technologie au sein des importations et exportations de l'OCDE implique que la production intérieure est devenue de plus en plus tributaire des technologies de pointe étrangères : les flux de R-D entre les pays devraient donc être retracés. Ces flux de R-D trans-frontières sont calculés en distinguant les sources de R-D par pays d'origine ainsi que les types de produits (biens intermédiaires et biens d'équipement).

\section{$R-D$ incorporée dans les achats de biens intermédiaires importés}

Pour un pays donné, la R-D incorporée dans les biens intermédiaires importés i achetés par le secteur $\mathrm{j}$ sera calculée en utilisant la formule : 


$$
\operatorname{RII}_{i j}=X_{i j}^{m_{i j}}\left(\sum_{k} \alpha_{i k} \cdot \frac{R_{i k}}{X_{i k}}\right)
$$

où $\mathrm{X}^{\mathrm{m}} \mathrm{ij}$ est la demande de consommation intermédiaire importée du secteur $\mathrm{j}$ concernant le produit $\mathrm{i}$ et a $\mathrm{ik}$ la part des importations du pays $\mathrm{k}$. Cet indicateur peut également être ventilé par pays d'origine de la R-D.

\section{$R-D$ incorporée dans les achats de biens d'équipements importés}

De la même manière, la R-D incorporée dans les biens d'équipement importés achetés par le secteur $\mathrm{j}$ au reste du monde peut être calculée par la formule :

$$
\left.R I N V^{m}{ }_{i j}=I N V^{m}{ }_{i j} \sum_{k} \alpha_{i k} \cdot \frac{R_{i k}}{X_{i k}}\right)
$$

\section{Total de la $R-D$ incorporée pour une industrie}

Enfin, le total des gains de R-D du secteur $\mathrm{j}$ peut être obtenu en additionnant les différents contenus en R-D indirecte par secteurs, et en ajoutant à cette somme les dépenses de R-D propres du secteur j lui même.

$$
R T_{j}=R_{j}+\sum_{i \neq j} R I I^{d} i j+\sum_{i \neq j} R I I_{i j}^{m_{i j}}+\sum_{i \neq j} R I N V_{i j}^{d}+\sum_{i \neq j} R I N V_{i j}^{m_{i j}}
$$

Dans l'équation ci-dessus, les éléments figurant sur les diagonales de chaque matrice sont éliminés afin d'éviter la double comptabilisation de la R-D propre et de l'autre R-D. Le premier terme de cette équation représente le montant de la R-D indirecte et les trois autres termes décrivent les mesures de la R-D indirecte incorporée dans les achats soit de biens intermédiaires soit de biens en capital effectués à l'intérieur et à l'étranger par le secteur $\mathrm{j}$. Le terme de ces indicateurs définissant l'intensité, à savoir la R-D incorporée par unité de production, peut être calculé simplement en divisant chaque terme de l'équation cidessus par la production du secteur $\mathrm{X}_{\mathrm{j}}$. D'autres indicateurs tels que le ratio entre la R-D directe et indirecte ou entre la R-D intérieure et importée ont aussi été calculés.

Bien que n'étant pas incluse dans cette note, l'approche utilisant la matrice des flux de technologie mentionnée ci-dessus peut être étendue pour incorporer les effets de propagation interindustriels en considérant les effets de second rang au moyen des flux de biens intermédiaires entre secteurs. L'inclusion de tels effets multiplicateurs dans le calcul de la R-D indirecte incorporée est évidemment justifiée. Par exemple, l'industrie des semi conducteurs entreprend des travaux de R-D à grande échelle. Les nouveaux modèles d'automobiles ou d'aéronefs utilisent de plus en plus les semi conducteurs pour le contrôle automatique des moteurs ou dans les systèmes de navigation avancés. Il est fréquent toutefois que ces secteurs n'achètent pas directement le semi conducteur qui est contenu dans une pièce détachée achetée à un fournisseur. Le modèle actuel ne prend pas en compte la R-D du semi conducteur incorporée dans ces pièces détachées lorsqu'il calcule la R-D indirecte utilisée par les industries automobile ou aéronautique. Le contenu précis en R-D de chaque produit peut seulement être estimé en utilisant l'approche de l'inverse de Leontief qui est présentée ci-dessous. 


\section{b) Approche inverse de la matrice de Leontief}

Les équations d'équilibre de la production brute dans un système d'entrées-sorties ouvert et statique des flux intérieurs peuvent s'écrire :

$$
X=A^{d} X+S^{d} I+F^{d}+E
$$

où $\mathrm{X}$ est le vecteur de la production brute, $\mathrm{A}^{\mathrm{d}}$ est la matrice des coefficients d'entrées sorties intérieurs, $\mathrm{S}^{\mathrm{d}}$ est la matrice de partage de l'investissement des entreprises privées, $\left(\mathrm{s}_{\mathrm{ij}} \mathrm{d}_{\mathrm{j}} \mathrm{I}_{\mathrm{ij}} / \mathrm{I}_{\mathrm{j}}\right.$ ) le vecteur de l'investissement total des entreprises privées par secteur d'origine, $\mathrm{F}^{\mathrm{d}}$ les vecteurs de la demande finale de produits intérieurs et d'importations à l'exclusion des dépenses d'investissement et $\mathrm{E}$ est le vecteur des exportations. Les dépenses d'investissement sont traitées comme exogènes dans l'équation (6).

A partir des équations de l'équilibre intérieur (6), on obtient :

$$
X=\left(I-A^{d}\right)^{-1}\left[S^{d} I+F^{d}+E\right]
$$

Si l'on définit l'intensité de R-D directe par secteur comme :

$$
r_{i}=\frac{R_{i}}{X_{i}} \quad(i=1,2, \ldots n)
$$

le vecteur du contenu en R-D totale intérieure $\mathrm{T}^{\mathrm{d}}$ peut être défini en pré multipliant les diagonales de la matrice des coefficients de R-D par secteur (8) par l'équation (7) pour obtenir :

$$
T^{d}=\hat{r}\left(I-A^{d}\right)\left[S^{d} I+F^{d}+E\right]
$$

L'équation (9) montre que le contenu en R-D intérieure totale peut être connecté à chaque composante de la demande intérieure finale et des exportations. A partir de l'équation (9), le contenu en $R$ - $D$ intérieure totale par unité de demande finale pour le secteur $j$ peut être défini par la somme des colonnes j de la matrice de coefficients ci-dessus :

$$
r f_{j}=\sum_{i=1}^{n} r_{i} b_{i j} \quad(j=1,2, \ldots, n)
$$

où $b_{i j}$ sont les élémens de l'inverse $B=\left(I-A^{d}\right)^{-i}$. Dès lors que la somme des colonnes $\mathrm{j}$ de l'inverse de Leontief B mesure le total des effets (directs et indirects) sur la production domestique lorsque la demande finale pour le jème secteur change d'une unité, la partie droite de l'équation (10) indique le contenu en R-D totale par unité de la livraison finale du produit $\mathrm{j}$.

Le calcul du contenu en R-D totale des biens intermédiaires achetés pour le secteur $\mathrm{j}$ est légèrement différent de l'équation (10) ci-dessus parce qu'il est basé sur la demande finale et non sur la production du secteur. Autrement dit, il concerne la quantité de R-D incorporée dans une unité de demande finale du produit $\mathrm{j}$ et non pas la quantité de R-D incorporée dans le produit $\mathrm{j}$. Le contenu en R-D du secteur doit répondre à la dernière question. Cette modification sera opérée en utilisant le multiplicateur produit par produit suivant : 


$$
\left[\begin{array}{cccc}
1-a_{11}^{d} & -a_{12}^{d} & \ldots & -a_{1, n-1}^{d} \\
-a_{21}^{d} & 1-a_{22}^{d} & \ldots & -a_{2, n-1}^{d} \\
: & : & : & : \\
-a_{n-1,1}^{d} & -a_{n-1,2}^{d} & \ldots & 1-a_{n-1, n-1}^{d}
\end{array}\right] \times\left[\begin{array}{c}
a_{1, n}^{d} \\
a_{2, n}^{d} \\
\vdots \\
a_{n-1, n-1}^{d}
\end{array}\right]=\left[\begin{array}{c}
\frac{b_{1, n}}{b_{n, n}} \\
\frac{b_{2, n}}{b_{n, n}} \\
\vdots \\
\frac{b_{n-1, n}}{b_{n, n}}
\end{array}\right]=B_{n}^{*}
$$

Les multiplicateurs ajustés ci-dessus indiquent un vecteur de la production totale de chaque secteur sauf le secteur $\mathrm{j}$ nécessaire pour produire une unité de produit pour l'industrie $\mathrm{n}$ (pour des raisons de commodité, faisons l'hypothèse que $n=j$ ). On définit donc la matrice des multiplicateurs ajustés sous la forme $\mathrm{B}^{*}=\left[\mathrm{B}_{1}, \mathrm{~B}_{2}, \ldots . ., \mathrm{B}_{\mathrm{n}}\right]$.

En utilisant les éléments de $\mathrm{B}^{*}$, on peut obtenir la R-D totale incorporée dans les biens intermédiaires d'origine intérieure destinés au secteur $\mathrm{j}$ en pré-multipliant le vecteur d'intensité de R-D directe comme :

$$
r i i_{j}{ }_{j}=\sum_{i \neq j}^{n-1} r_{i j} b_{i j}^{*}
$$

Les autres formules de contenu en R-D correspondant aux équations (2) à (5) seront définies de la manière suivante.

* R-D incorporée dans les biens d'équipement d'origine intérieure achetés pour le secteur $j$

$$
r i n v_{j}^{d}=\sum_{i=1}^{n} r_{i}\left(\sum_{k=1}^{n} b_{i k} s_{k j}{ }_{k j}\right)
$$

* R-D incorporée dans les biens intermédiaires importés achetés pour le secteur $j$ ( $k$ indique le pays)

$$
r i i_{j}^{m}=\sum_{i=1}^{n} a_{i j}^{m} \sum_{k=1}^{n}\left(\alpha_{i k} \cdot r f_{i k}^{d}\right)
$$

* $R$-D incorporée dans les biens d'équipement importés achetés pour le secteur j( $k$ indique le pays)

$$
\operatorname{rinv}_{j}{ }_{j}=\sum_{i=1}^{n} s_{i j}^{m} \sum_{k=1}^{n}\left(\alpha_{i k} \cdot \operatorname{tinv}^{d}{ }_{i k}\right)
$$

* R-D totale incorporée pour le secteur $j$

$$
r t_{j}=r_{j}+\operatorname{rid}_{j}{ }_{j}+\operatorname{rinv}^{d}{ }_{j}+\operatorname{rii}^{m}{ }_{j}+\operatorname{rinv}^{m}{ }_{j}
$$




\section{Annexe 2}

Tableau 3. Classification des industries selon leur intensité technologique (CITI Révision 2) Tableau 4. Liste des produits de haute technoogie (CTCI Révision 3) 


\section{Tableau 3. Classification des industries selon leur intensité technologique (CITI Révision 2)}

\begin{tabular}{|c|c|c|c|c|c|c|c|}
\hline & \multirow[b]{2}{*}{ CITI Rév.2 } & \multicolumn{3}{|c|}{1990} & \multicolumn{3}{|c|}{1980} \\
\hline & & $\begin{array}{c}\text { R-D globale } \\
\text { production }\end{array}$ & $\underline{\mathrm{R}-\mathrm{D}}$ & $\begin{array}{l}\frac{\mathrm{R}-\mathrm{D}}{\text { Valeur }} \\
\text { ajoutée }\end{array}$ & $\begin{array}{c}\text { R-D } \\
\text { globale } \\
\text { production }\end{array}$ & $\frac{\mathrm{R}-\mathrm{D}}{\text { production }}$ & $\begin{array}{l}\frac{\mathrm{R}-\mathrm{D}}{\text { Valeur }} \\
\text { ajoutée }\end{array}$ \\
\hline \multicolumn{8}{|c|}{ Industries de haute technologie } \\
\hline Construction aéronautique & 3845 & 17.30 & 19.48 & 36.25 & 16.06 & 14.13 & 41.11 \\
\hline Machines de bureau et à calculer & 3825 & 14.37 & 11.46 & 30.49 & 11.19 & 9.00 & 26.01 \\
\hline Produits pharmaceutiques & 3522 & 11.35 & 10.47 & 21.57 & 8.37 & 7.62 & 16.89 \\
\hline Appareil radio, TV et télécomm. & 3832 & 9.40 & 8.03 & 18.65 & 9.33 & 8.35 & 18.43 \\
\hline \multicolumn{8}{|c|}{ Industries de moyenne-haute technologie } \\
\hline Instruments scientifiques & 385 & 6.55 & 5.10 & 11.19 & 4.69 & 3.61 & 8.63 \\
\hline Véhicules automobiles & 3843 & 4.44 & 3.41 & 13.70 & 3.68 & 2.81 & 10.05 \\
\hline Machines et appareils électriques & $383-3832$ & 3.96 & 2.81 & 7.63 & 4.25 & 3.48 & 8.85 \\
\hline Industries chimiques & $\begin{array}{l}351+352+ \\
3522\end{array}$ & 3.84 & 3.20 & 8.96 & 2.67 & 2.15 & 7.60 \\
\hline Autres matériels de transport & $\begin{array}{l}3842 \\
+3844 \\
+3849\end{array}$ & 3.03 & 1.58 & 3.97 & 1.69 & 0.98 & 2.70 \\
\hline Machines non électriques & $382-3825$ & 2.58 & 1.74 & 4.58 & 2.00 & 1.32 & 3.48 \\
\hline \multicolumn{8}{|c|}{ Industries de moyenne-faible technologie } \\
\hline Caoutchouc et matières plastiques & $355+356$ & 2.47 & 1.07 & 3.02 & 2.20 & 1.08 & 3.27 \\
\hline Construction navale & 3841 & 2.21 & 0.74 & 2.13 & 1.42 & 0.39 & 1.11 \\
\hline Autres industries manufacturières & 39 & 1.76 & 0.63 & 1.52 & 1.45 & 0.79 & 1.19 \\
\hline Métaux non ferreux & 372 & 1.57 & 0.93 & 3.48 & 1.04 & 0.54 & 2.29 \\
\hline Produits minéraux non métalliques & 36 & 1.44 & 0.93 & 2.20 & 1.10 & 0.66 & 1.72 \\
\hline Ouvrages en métaux & 381 & 1.35 & 0.63 & 1.39 & 1.06 & 0.45 & 1.08 \\
\hline Raffinage du pétrole & $353+354$ & 1.33 & 0.96 & 8.43 & 0.80 & 0.58 & 6.17 \\
\hline Métaux ferreux & 371 & 1.10 & 0.64 & 2.48 & 0.78 & 0.45 & 1.71 \\
\hline \multicolumn{8}{|c|}{ Industries de faible technologie } \\
\hline Papier, imprimerie et édition & 34 & 0.88 & 0.31 & 0.76 & 0.68 & 0.23 & 0.61 \\
\hline Textiles, habillement et cuir & 32 & 0.78 & 0.23 & 0.65 & 0.56 & 0.13 & 0.38 \\
\hline Alimentation, boissons et tabac & 31 & 0.73 & 0.34 & 1.14 & 0.56 & 0.23 & 0.93 \\
\hline Bois et meubles & 33 & 0.65 & 0.18 & 0.47 & 0.55 & 0.14 & 0.39 \\
\hline
\end{tabular}

Source : OCDE, bases de données ANBERD, STAN, Entrées-sorties et BTD (DSTI, Division AES) 
Tableau 4. Liste des produits de haute technologie - CTCI Révision 3

(Période 1988-95)

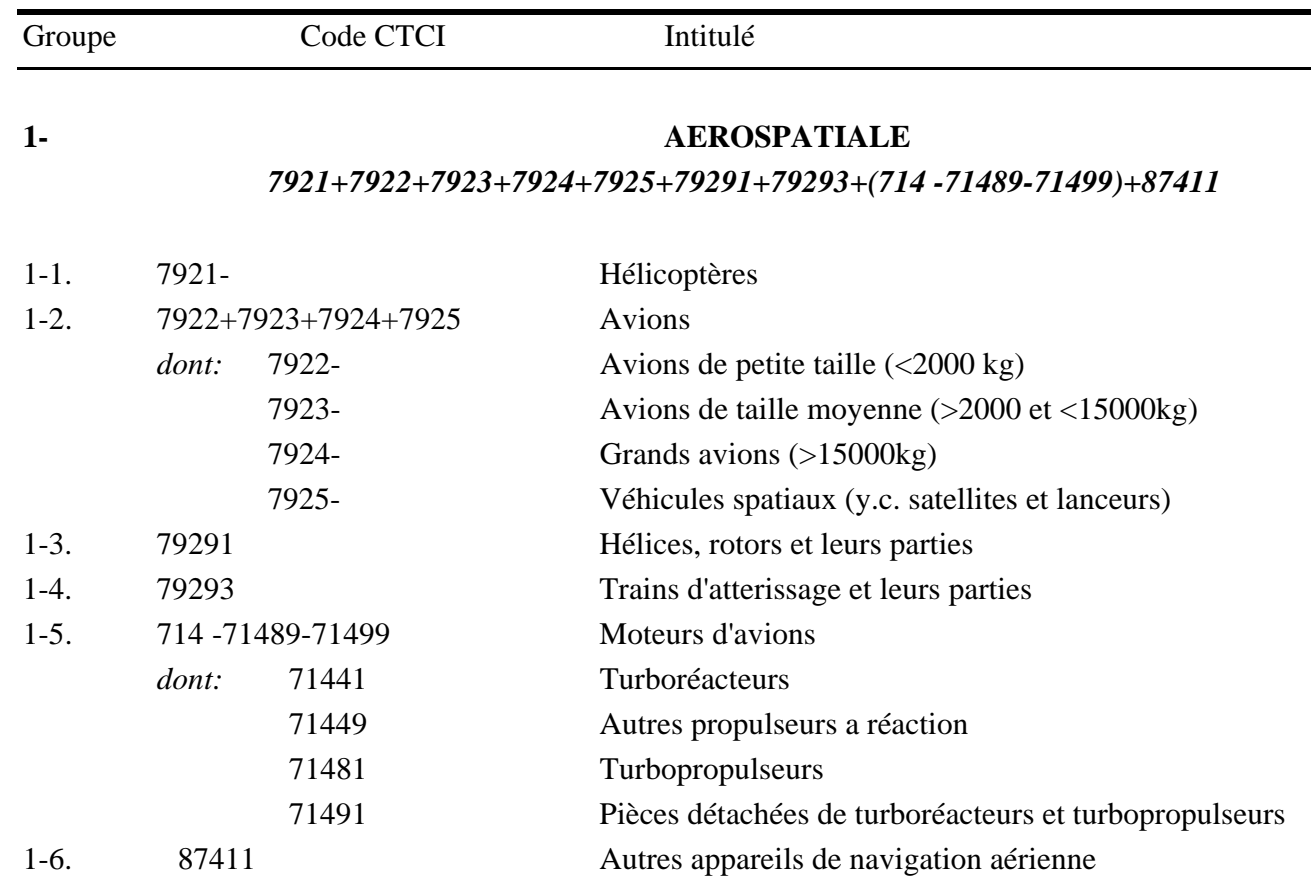

2-

ORDINATEURS - MACHINES DE BUREAU

$75113+75131+75132+75134+(752-7529)+75997$

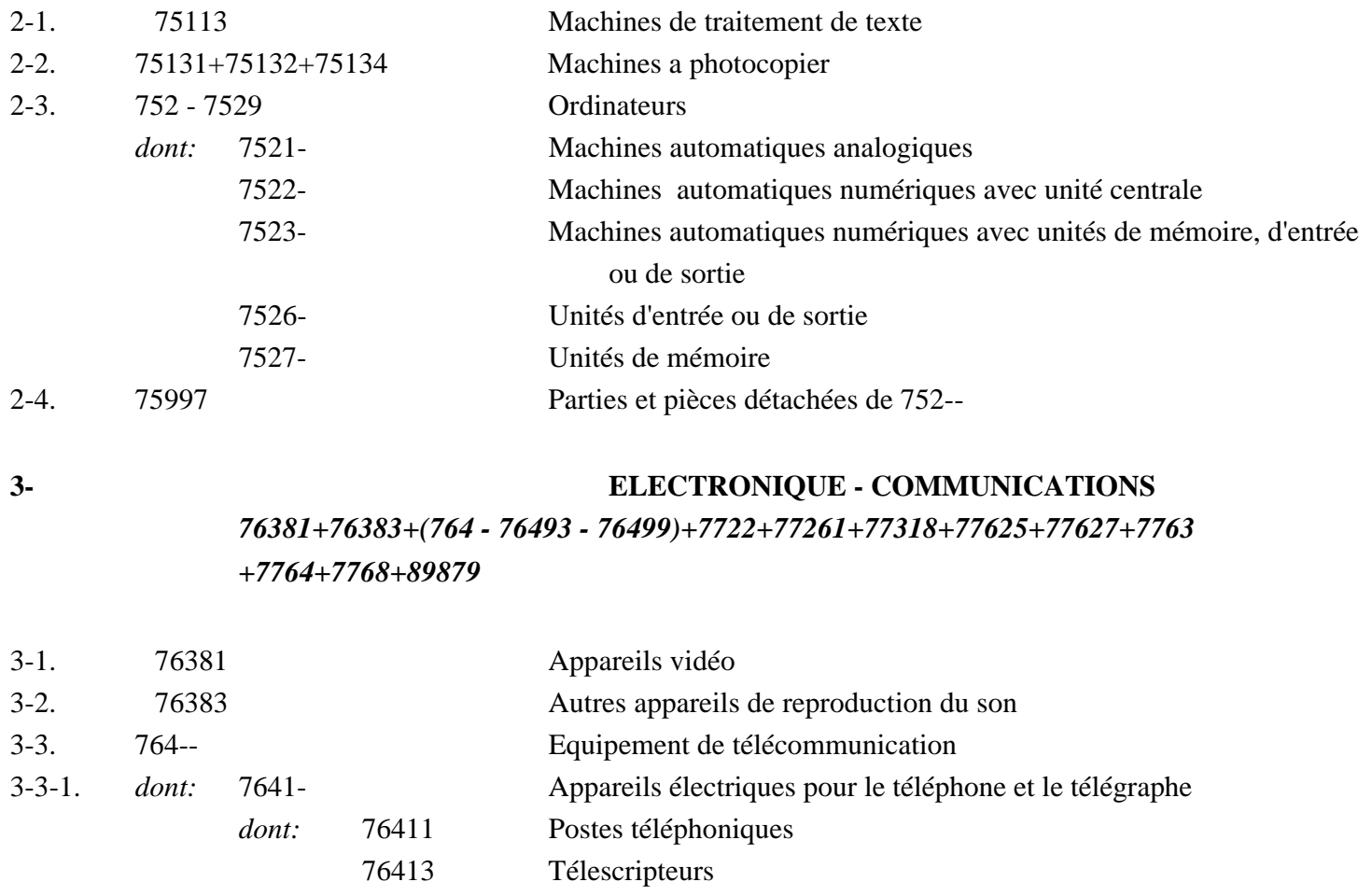




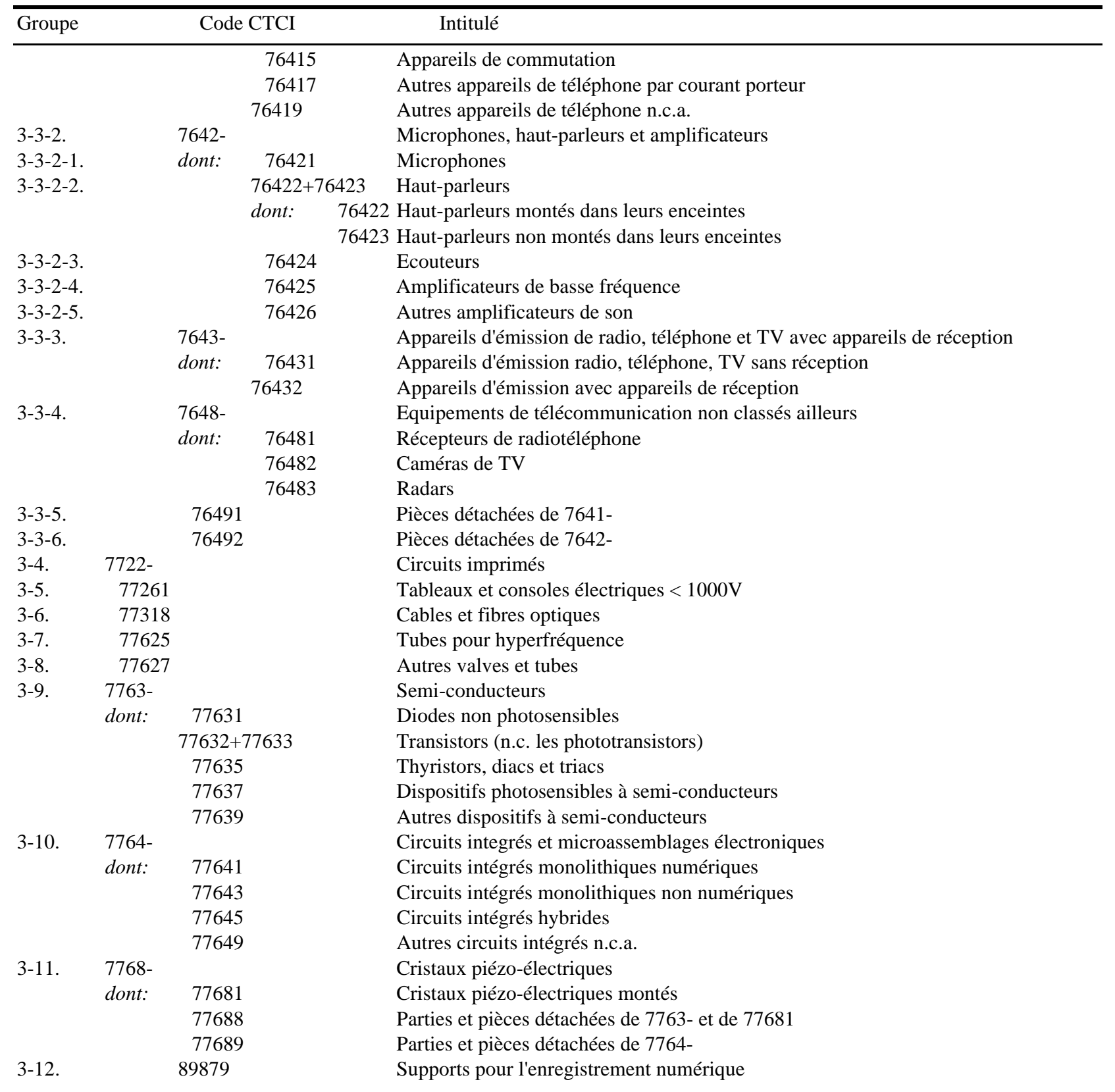

4-

PHARMACIE

$5413+5415+5416+5421+5422$

$\begin{array}{lll}\text { 4-1. 5413- } & & \text { Antibiotiques } \\ \text { dont: } & 54131 & \text { Penicillines et leurs derivés } \\ & 54132 & \text { Streptomycines } \\ & 54133 & \text { Tétracyclines et leurs dérivés }\end{array}$




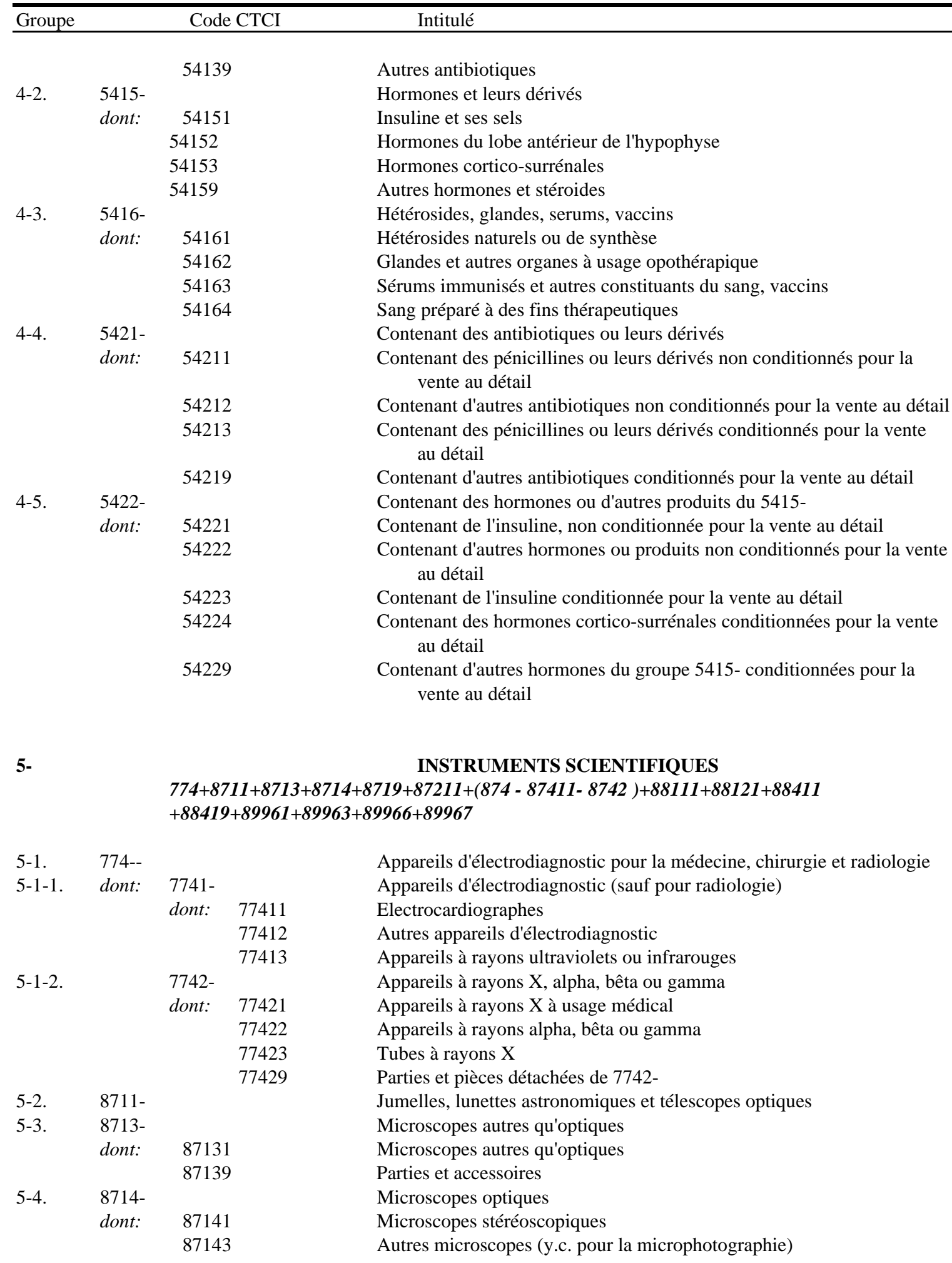




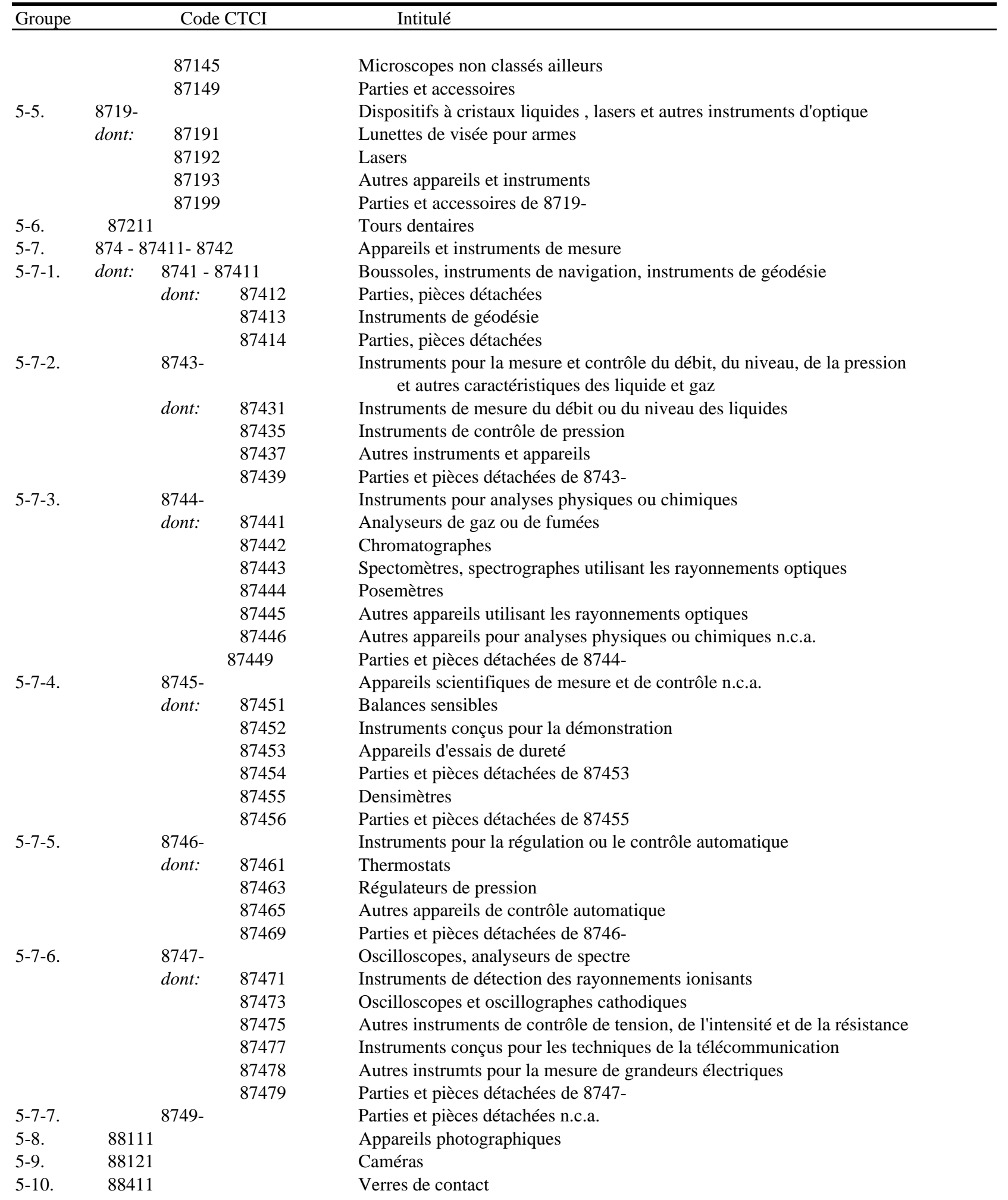




$\begin{array}{lcl}\text { 5-11. } & 88419 & \text { Fibres optiques autres que ceux du groupe 7731- } \\ 5-12 . & 89961 & \text { Appareils de prothèse auditive } \\ 5-13 . & 89963 & \text { Appareils d'orthopédie } \\ 5-14 . & 89966 & \text { Appareils de prothèse oculaire } \\ \text { 5-15. } & 89967 & \text { Stimulateurs cardiaques }\end{array}$

6-

$77862+77863+77864+77865+7787+77884$

$\begin{array}{llll}\text { 6-1. } & \text { 77862+77863+77864+77865 } & \text { Condensateurs électriques fixes } \\ & \text { dont: } & 77862 & \text { Condensateurs fixes ou tantales } \\ & 77863 & \text { Condensateurs fixes électrolytiques à l'aluminium } \\ & 77864 & \text { Condensateurs fixes à diélectrique en céramique à une seule couche } \\ & & \text { Condensateurs fixes à diélectrique en plusieurs couches } \\ & & \text { Machines électriques ayant une fonction propre } \\ \text { 6-2. } & 7787- & & \text { Accélérateurs de particules } \\ & \text { dont: } & 77871 & \text { Autres machines ayant une fonction propre } \\ & 77878 & \text { Parties et pièces détachées de } 7787- \\ & & 77879 & \text { Appareils de signalisation acoustique ou visuelle }\end{array}$

7-

\section{CHIMIE}

$52222+52223+52229+52269+525+531+57433+591$

\begin{tabular}{|c|c|c|c|c|}
\hline \multirow[t]{5}{*}{ 7-1. } & \multicolumn{3}{|c|}{$52222+52223+52229+52269$} & Produits inorganiques \\
\hline & dont: & 52222 & & Sélénium, tellure, phosphore, arsenic et bore \\
\hline & & 52223 & & Silicium \\
\hline & & 52229 & & Calcium, strontium et baryum \\
\hline & & 52269 & & Autres bases inorganiques \\
\hline 7-2. & 525-- & & & Matières radioactives \\
\hline $7-2-1$. & dont: & $5251-$ & & Isotopes radioactifs \\
\hline & & dont: & 52511 & Uranium naturel et ses composants \\
\hline & & & 52513 & Uranium enrichi en U235, plutonium et ses composés \\
\hline & & & 52515 & Uranium appauvri en U235 \\
\hline & & & 52517 & Combustibles des réacteurs nucléaires \\
\hline & & & 52519 & Isotopes radioactifs n.c.a. \\
\hline $7-2-2$. & & $5259-$ & & Isotopes stables et leurs composés \\
\hline & & dont: & 52591 & Isotopes autres que 5251- \\
\hline & & & 52595 & Composés inorganiques ou organiques des métaux des terres rares \\
\hline $7-3$. & $531--$ & & & Colorants organiques \\
\hline & dont: & $5311-$ & & Matières colorantes organiques \\
\hline & & $5312-$ & & $\begin{array}{l}\text { Produits organiques synthétiques utilisés comme agents d'avivage } \\
\text { fluorescents ou luminophores }\end{array}$ \\
\hline 7-4. & 57433 & & & Polyéthylène térephtalate \\
\hline $7-5$. & 591-- & & & Insecticides, désinfectants \\
\hline & dont: & $5911-$ & & Insecticides \\
\hline & & $5912-$ & & Fongicides \\
\hline & & $5913-$ & & Herbicides, inhibiteurs de germination \\
\hline & & 5914- & & Désinfectants \\
\hline
\end{tabular}


Intitulé

\section{MACHINES NON ELECTRIQUES}

$71489+71499+71871+71877+71878+72847+7311+73131+73135+73142+73144+$

$73151+73153+73161+73163+73165+73312+73314+73316+7359+73733+73735$

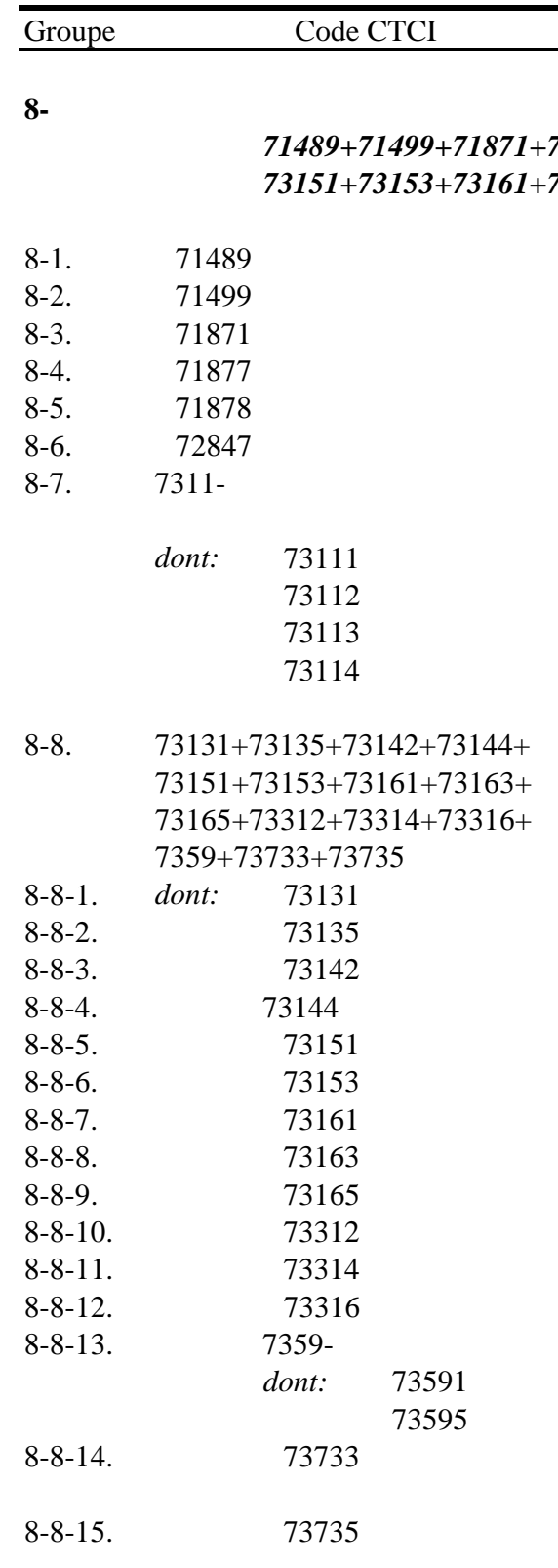
9- 891--
9-1. 8911-
9-2. 8912-
9-3. $\quad 8913$
9-4. $\quad$ 8919-

Autres turbines à gaz

Pièces détachées de turbines à gaz

Réacteurs nucléaires

Eléments combustibles non irradiés

Parties de réacteurs nucléaires

Machines et appareils pour la séparation isotopique

Machines-outils opérant par laser ou autres faisceaux de lumière, de photons, ultrasons, électro-érosion

Opérant par laser ou autres faisceaux de lumière ou de photons

Opérant par ultra-son

Opérant par électro-érosion

Opérant par procédés électrochomiques, par faisceaux d'électrons, ioniques ou par jet de plasma

Machines-outils à commande numérique

Tours horizontaux à commande numérique

Autres tours à commande numérique

Autres machines à percer à commande numérique

Autres aliseuses-fraiseuses à commande numérique

Machines à fraiser, à console à commande numérique

Autres machines à fraiser à commande numérique

Machines à rectifier les surfaces à commande numérique

Autres machines à rectifier à commande numérique

Machines à affûter à commande numérique

Machines à rouler, cintrer, plier à commande numérique

Machines à cisailler à commande numérique

Machines à poinçonner à commande numérique

Pièces détachées de 731-- et 733--

Parties et pièces détachées de 731--

Parties et pièces détachées de 733--

Machines pour le sondage des métaux par résistance totalement ou partiellement automatiques

Machines pour le sondage des métaux au jet de plasma totalement ou partiellement automatiques

\section{ARMEMENT}

Chars et automobiles blindés de combat

Bombes, torpilles, mines, missiles, etc...

Armes autres que de guerre (engins pour lancer des fusées de signalisation...)

Parties et pièces détachées de 89112, 89114 et 8913 


\section{NOTES}

1. "Commerce international de produits de haute intensité de recherche-développement" [STIC/80.48]. "Le commerce international des produits de haute technologie : une approche empirique" (mémorandum interne à l'OCDE).

2. "Spécialisation et compétitivité des industries manufacturières en haute, moyenne et faible intensité de R-D” : “Tendances générales" (mémorandum interne à l'OCDE).

3. Les données de R-D utilisées proviennent de la Base de données ANBERD de l'OCDE. Cette base de données analytiques sur les dépenses de recherche et de développement dans les secteurs des entreprises est élaborée à partir d'estimations visant à obtenir un ensemble de données cohérentes qui pallie les problèmes de comparabilité internationale et de discontinuité temporelle associés aux données officielles de R-D du secteur des entreprises fournies par les pays Membres de l'OCDE. ANBERD contient les dépenses de R-D pour la période 1973-95 par industrie et pour 15 pays de l'OCDE.

4. Il s'agit des secteurs pour lesquels on dispose de données complètes et des pays pour lesquels des tableaux input-output harmonisés sont disponibles : Etats-Unis, Japon, Allemagne, France, Royaume-Uni, Italie, Canada, Australie, Pays-Bas et Danemark.

5. Néanmoins, il faut reconnaître que les classifications même au niveau de produits ne sont pas suffisamment détaillées pour classer dans des catégories différentes des produits qui portent le même nom mais appartiennent à des gammes très différentes.

6. Une matrice de conversion entre CTCI Révision 3 (classification par produit) et CITI Révision 2 (classification par secteur industriel) a été utilisée à cette fin.

7. La Base de données de l'OCDE sur les entrées-sorties contient les matrices de biens intermédiaires et d'investissement (domestiques et importées) pour des années sélectionnées dans la période 1970-90. Elle couvre dix pays Membres de l'OCDE et englobe 36 industries dont 22 du secteur manufacturier. 


\section{DOCUMENTS DE TRAVAIL DE LA DSTI}

\section{6}

1. Embodied Technology Diffusion: An Empirical Analysis for 10 OECD Countries George Papaconstantinou, Norihisa Sakurai and Andrew Wyckoff

2. The Impact of R\&D and Technology Diffusion on Productivity Growth: Evidence for 10 OECD Countries in the 1970s and 1980s

Norihisa Sakurai, Evangelos Ioannidis and George Papaconstantinou

3. Short-term Indicators: Using Qualitative Indicators to Update Production Indices

Paul Schreyer and Corinne Emery

4. SMEs and Employment Creation: Overview of Selected Quantitative Studies in OECD Member Countries

Paul Schreyer

5. Globalisation and Competitiveness: Relevant Indicators

Thomas Hatzichronoglou

6. Factors Influencing the Steel Work Force: 1990 to 1995

Donald F. Barnett

7. Measuring R\&D in the Services

Alison Young

8. The Evolution of Skills in OECD Countries and the Role of Technology

A. Colecchia and G. Papaconstantinou

1997

1. Bibliometric Indicators and Analysis of Research Systems: Methods and Examples

(disponible en français sous le titre "Indicateurs bibliométriques et analyse des systèmes de recherche : méthodes et exemples")

Yoshiko Okubo

2. Revision of the High-technology Sector and Product Classification

(disponible en français sous le titre "Révision des classifications des secteurs et des produits de haute technologie")

Thomas Hatzichronoglou 